\section{ALFRED RUSSEL WALLACE, ANTROPÓLOGO: CONTRIBUCIONES A LA ANTROPOLOGÍA FÍSICA}

\author{
Juan Manuel Rodríguez Caso \\ Universidad Nacional Autónoma de México \\ ORCID iD: http://orcid.org/0000-0003-0745-4657 \\ carcharhinus_7@yahoo.com
}

\section{ALFRED RUSSEL WALLACE, ANTHROPOLOGIST: CONTRIBUTIONS TO PHYSICAL ANTHROPOLOGY}

Cómo citar este artículo/Citation: Rodríguez Caso, J. M. (2020). Alfred Russel Wallace, antropólogo: contribuciones a la antropología física. Arbor, 196 (797): a565. https://doi. org/10.3989/arbor.2020.797n3002
Copyright: (C) 2020 CSIC. Este es un artículo de acceso abierto distribuido bajo los términos de la licencia de uso y distribución Creative Commons Reconocimiento 4.0 Internacional (CC BY 4.0).
RESUMEN: La figura de Alfred Russel Wallace dentro de la antropología ha sido escasamente reconocida dentro de la historia de la disciplina. Un punto importante es ubicar al autor dentro de las discusiones antropológicas de la época victoriana, desde su interés temprano como naturalista por historizar a los seres humanos, hasta su labor institucional como el primer presidente de un departamento de antropología en Inglaterra. En ese contexto, el objetivo del trabajo es presentar la visión de Wallace respecto a la antropología física, a partir de su obra antropológica, que incluye referencias al origen de las razas humanas en términos naturalistas, al desarrollo de características físicas distintivas de los seres humanos o a la relación evolutiva entre primates. Con esto, se amplía la perspectiva sobre el desarrollo de la antropología física en el siglo XIX, al abrir el abanico a autores que contribuyeron de manera importante a su desarrollo.

PALABRAS CLAVE: Alfred Russel Wallace; evolución; antropología física; naturalismo; instituciones científicas.
ABSTRACT: The figure of Alfred Russel Wallace as an anthropologist has scarcely been recognised in the history of the discipline. An important point is to place the author within the anthropological discussions of the Victorian era, from his early interest as a naturalist in historicizing human beings, to his institutional work as the first president of an anthropology department in England. In this context, the paper seeks to present Wallace's perspective on physical anthropology, based on his anthropological work, which includes references to the origin of human races in naturalistic terms, the development of distinctive physical characteristics of human beings, or the evolutionary relationship between primates. With this, perspective on the development of physical anthropology in the nineteenth century is broadened, opening up the spectrum to authors who contributed significantly to its development.

KEYWORDS: Alfred Russel Wallace; Evolution; Physical Anthropology; Naturalism; Scientific Institutions. 


\section{INTRODUCCIÓN}

Charles Darwin (1809-1882), contrariado, cerraba una carta en enero de 1870 , con un fatídico mensaje: "But I groan over Man- you write like a metamorphosed (in retrograde direction) naturalist, \& you the author of the best paper that ever appeared in Anth. Review! Eheu Eheu Eheu | Your miserable friend | C. Darwin" (http://www.darwinproject.ac.uk/DCP-LETT-7086). El destinatario no era otro sino su amigo, el naturalista Alfred Russel Wallace (1823-1913). La relación entre ambos personajes fue mucho más allá del conocido momento de la publicación conjunta de la teoría de la selección natural en 1858 , pues se puede hablar de una amistad que duró casi 25 años, fácilmente apreciable en el continuo intercambio de correspondencia entre ambos. Uno de los tópicos de discusión más frecuente entre ambos fue el hombre, el ser humano, que al menos durante la década de 1860 fue posiblemente el tema más recurrente entre los naturalistas británicos.

La respuesta de Darwin hace relación a una de las obras más importantes escritas por Wallace sobre temas antropológicos, The Origin of Human Races and the Antiquity of Man Deduced From the Theory of «Natural Selection», un trabajo presentado el $1 .{ }^{\circ}$ de marzo de 1864 en una reunión de la Anthropological Society of London (ASL), y publicado posteriormente el mismo año en la revista de la sociedad ${ }^{1}$. Es el primer escrito en que explícitamente se utiliza el principio de la selección natural para explicar el origen y la diversidad de los seres humanos, una cuestión poco reconocida por los historiadores en general, pero como lo ha señalado el historiador de la antropología George W. Stocking: "Wallace's flash of insight [on ethnography and evolution] helped to transform the terms in which speculation on racial diversity was carried on" (Stocking, 1987, p. 101).

Hablar de Wallace y la evolución humana remite tradicionalmente en términos históricos a su propuesta sobre los límites de la selección natural en el origen y desarrollo de las capacidades mentales. No obstante, como se verá a lo largo de este artículo, desde su temprano interés en la transmutación de los seres humanos en diciembre de 1845 (McKinney, 1969), su involucramiento en la antropología fue sumamente amplio, y es que como han hecho ver historiadores como Henry Lewis McKinney, ese interés inicial fue la base a partir de la cual Wallace desarrolló su visión de la selección natural (McKinney, 1972). En general, se ha destacado el marco filosófico desde el que terminó por concebir el estudio de lo humano en términos "naturales", este último un concepto tan amplio que lo mismo incluía la teoría de la selección natural, el espiritismo o el socialismo, todo ello concebido como extensiones "naturales" de un proceso evolutivo teleológico (Smith, 1972). Un aspecto controversial dentro de la obra de Wallace fue el espiritismo, en la medida en que ha sido considerado como el "punto de partida" respecto a otros hombres de ciencia victorianos, aunque en términos del contexto de la época, el espiritismo fue un movimiento amplio e influyente que proporcionó a personajes como Wallace elementos alternativos para explicar la realidad, como fue la concepción de la "naturaleza espiritual" de la materia (Kottler, 1974), una postura que reafirmó con el paso de los años a partir de un mayor involucramiento en comprender el cerebro humano (Eiseley, 1959). Tal vez uno de los aspectos más llamativos en los que Wallace basó su propuesta de los límites de la acción de la selección natural fueron las cualidades estéticas y musicales, al considerar de la misma manera que muchos de sus contemporáneos victorianos que esas características eran exclusivas del mundo "civilizado" (Brotman, 2001). Un punto importante es recordar la importancia de los viajes que realizó en el sentido etnográfico, pues le permitieron tener información de primera mano a partir de la cual reflexionar sobre el origen y la historia de los seres humanos, que incluso sirve para caracterizar a Wallace como un antropólogo (Ellen, 2011; Rodríguez Caso, 2015; Rodríguez Caso, 2019; Vetter, 2009).

A partir de lo anterior, este artículo se centra en: primero, ubicar a Wallace en el contexto de las discusiones sobre evolución humana de la época victoriana, inicialmente desde sus esfuerzos por "historizar" a los seres humanos en términos evolutivos, para con ello resaltar posteriormente un aspecto de su vida profesional muy poco destacado (incluso por él mismo), como fue su participación en instituciones dedicadas a discusiones antropológicas. Y segundo, mostrar que su interés en la evolución humana se puede ver reflejado a través de una serie de discusiones relacionadas con la antropología física, que abarcan desde el caso ya mencionado sobre el origen y diversificación de las razas humanas hasta las relaciones evolutivas entre primates y seres humanos, pasando por el desarrollo de características distintivas de lo humano. 


\section{WALLACE Y SU LUGAR EN LA ANTROPOLOGÍA VICTORIANA}

\section{a. Historizando a los seres humanos}

Como se mencionó anteriormente, el interés de Wallace por entender la transmutación de los seres humanos se dio de manera paralela al reconocimiento inicial del cambio en los organismos como un hecho. A partir de una carta que Wallace envía a su amigo, el entomólogo Henry W. Bates (1825-1892) el 28 de diciembre de 1845, aclara su convencimiento del argumento transmutacionista planteado en el controvertido Vestiges of the Natural History of Creation (1844) (McKinney, 1969, p. 372), publicado originalmente de manera anónima por el periodista escocés Robert Chambers, y que, en el contexto victoriano, presentó de manera pública una propuesta descriptiva de la evolución del universo y de las estrellas, así como un proceso de transmutación progresiva de las especies. Fue una obra que tuvo una positiva recepción entre el público en general (Secord, 2000), pero fue enormemente cuestionada por los hombres de ciencia de la época, con muy contadas excepciones, como fue el caso de Wallace. Además de aceptar la evolución, el otro punto destacable de la carta a Bates tiene que ver con entender ese proceso de transformación a partir del ejemplo de los seres humanos, inspiración tomada de la obra de los médicos James C. Prichard (1786-1848) y WiIliam Lawrence (1783-1867), quienes más que hablar propiamente de una transmutación de las razas humanas, retoman la idea de un origen común y de un cierto proceso de desarrollo de características distintivas de los individuos (McKinney, 1969, p. 372).

A manera de aclaración, dados los diversos usos que puede tener el término historizar, es importante puntualizar que, en el caso de Wallace, es hablar de un "proceso de construcción de historias humanas con diversos fines (científicos, religiosos y sociopolíticos)", en los que destacan "sus contribuciones a los debates sobre el origen de las razas humanas" (Rodríguez Caso, 2019, p. 143). Además, esta visión de la historia de los seres humanos era compartida por otros naturalistas de la época, como Charles Darwin y Charles Lyell, en el sentido de valorar que la práctica científica ya no estaba enfocada en lo estático, sino que se privilegió el estudio de los procesos de cambio en la naturaleza (Davis, 1990, p. 57).

Ese interés en los seres humanos se extendió a buena parte de la obra de Wallace. De hecho, su carrera como naturalista se dio de manera paralela a su interés en comprender la naturaleza de los seres humanos. Una parte fundamental de esa carrera fue la experiencia ganada a través de los viajes que realizó, tanto al Amazonas (1848-1852) como al archipiélago Malayo (1854-1862), en los cuales tuvo numerosas experiencias que hicieron que ganara en conocimiento no solamente sobre las costumbres de los diversos grupos con los que interactuó, sino que también le plantearon dudas respecto a ese interés inicial por buscar la comprensión del origen de las razas humanas y su posterior transformación a lo largo del tiempo. Trabajos como "Notes of a Journey Up the Sadong River, North-West Borneo", publicado en Proceedings of the Royal Geographical Society of London en 1856, presentan por un lado información geográfica, y por el otro, una descripción de los habitantes de la región. La descripción que hace Wallace tanto de los Dyaks como de los malayos que habitaban la zona incluía el tipo de vestimenta, el chawat, una tela de algodón que se amarra a la cintura, que se podía completar con un pañuelo en la cabeza. Sobre aspectos físicos de los humanos, Wallace resalta:

It is highly curious that countries so distant as Borneo and the valley of the Amazon, between which we can by no possibility imagine any direct communication to have ever taken place, should yet contain indigenes so similar to each other; for between some tribes of Dyaks and of Amazon Indians, I can call to mind no one physical distinction" (Wallace, 1856a, p. 203).

Esta afirmación es un ejemplo de cómo a partir de las observaciones en el campo, Wallace empezó muy pronto a considerar la importancia de la cultura en el desarrollo de las sociedades. A mediados del siglo XIX era común encontrar estudios que conjuntaban tanto la geografía como la etnología, en la medida en que esa información era vital para el imperio británico.

Otro ejemplo de ese abordaje etnográfico fue el artículo resultado de su experiencia en Nueva Guinea. Como en otras publicaciones, además de incorporar descripciones geográficas y geológicas, dedica espacio a caracterizar a los papúas: "the flat forehead, heavy brows, and large nose, with the apex bent downwards, are almost universal, as well as the harsh curly hair, which often forms an enormous stiff mop, and is then highly esteemed" (Wallace, 1860, p. 174), así como a hablar de lo que considera características mentales y morales: "They are much more impulsive, and do not conceal their emotions and passions. They are inquisitive, talk much and loudly, and laugh boisterously..." (Wallace, 1860, p. 174). 
El trabajo de Wallace sobre los seres humanos fue llevado de la mano, por un lado, de las observaciones que pudo realizar durante sus viajes, y por el otro, del afán en construir una "filogenia de las diferentes razas humanas para afirmar un origen común para todas ellas" (Rodríguez Caso, 2019, p. 144). Aunque sus primeras experiencias etnográficas en Gales, durante su estancia como topógrafo en su juventud (1840-1843), no suelen ser consideradas de particular relevancia en su formación como naturalista, fue en ese tiempo en el que colectó información a partir de la cual realizaría uno de sus primeros escritos, "The South Wales Farmer", que sería publicado posteriormente en su autobiografía, en 1905 (Wallace, 1905, vol. I, pp. 206-222). Este tipo de trabajos se pueden caracterizar como etnográficos, y con un profundo interés en aspectos culturales, todo dentro de una propuesta histórica.

Su perspectiva histórica se vio reforzada por la importante influencia del trabajo de Charles Lyell, que como a otros autores (como el mismo Charles Darwin) proporcionó un marco explicativo de la historia, no solamente del ser humano sino de la naturaleza en general, a partir del tiempo profundo ${ }^{2}$. Es tal la importancia de la historia para Lyell, que "[I]a ciencia geológica [...] o es histórica o no es ciencia" (Álvarez Muñoz, 2004 , p. 112). Fue esa importancia del aspecto histórico la que fue retomada por Wallace en obras como el conocido Ensayo Sarawak ("On the Law Which Has Regulated the Introduction of New Species", 1855), en "On the Natural History of the Aru Islands" (1857) o en "On the Zoological Geography of the Malay Archipelago" (1859), para sustentar con ello una propuesta de explicación del origen y ramificación de las especies a lo largo del tiempo (Hodge, 2015, pp. 59-60). Fue la experiencia de sus viajes la que le proporcionó a Wallace una nueva perspectiva sobre la historización de los seres humanos, ya que a partir de ahí se interesó por los estudios lingüísticos, y posteriormente, como parte de su búsqueda por un mecanismo que explicara la transmutación de las especies, por el caso concreto del origen de las razas humanas (Rodríguez Caso, 2019, p. 151-164). Vale recordar aquí, como lo ha señalado Michael Bravo, que: "Comparative studies of mythology and language helped to breed a measure of cultural relativism. Differences in language and vocabularies were used to account for diversity, while similarities were taken as evidence for the unity of humanity" (Bravo, 1996, p. 339). A partir de esto, y como se verá más adelante con el caso del lenguaje, Wallace fue capaz de afirmar que la selección natural no era suficiente para explicar características humanas como la mente (Wallace, 1869a), una expli- cación basada en una férrea defensa del utilitarismo, o principio de utilidad, que irónicamente fue tomado de la obra más conocida de Charles Darwin, El origen de las especies (Fichman, 2004, p. 192), quien decía:

Natural selection will never produce in a being anything injurious to itself, for natural selection acts solely by and for the good of each. No organ will be formed, as Paley has remarked, for the purpose of causing pain or for doing an injury to its possessor. If a fair balance be struck between the good and evil caused by each part, each will be found on the whole advantageous. After the lapse of time, under changing conditions of life, if any part comes to be injurious, it will be modified; or if it be not so, the being will become extinct, as myriads have become extinct.

(Darwin, 1859, p. 201)

Esto llevó a Wallace a la controvertida conclusión que presentó sobre todo en su reseña de 1869 sobre las obras de Lyell, al mantener que bajo el principio de utilidad la selección natural no era la responsable del surgimiento de características humanas como el cerebro, los órganos del lenguaje, la mano, y la forma externa del cuerpo (Fichman, 2004, p. 192). Como veremos más adelante, su visión de la antropología era mucho más amplia, al punto de interesarse no solamente en explicaciones físicas sobre lo humano, sino también en los aspectos culturales.

El interés de Wallace en historizar a los seres humanos terminó por ser el eje de su labor como naturalista, en la medida en que no solamente se centró en buscar explicaciones propiamente naturalistas a partir de sus observaciones en el campo, sino también en la teorización, situación que se puede ver reflejada no solamente en sus publicaciones sino, como veremos, en su participación dentro de las instituciones científicas dedicadas a temas antropológicos y etnológicos.

\section{b. Su participación institucional}

Una de las grandes lagunas en la vida de Wallace ha sido su lugar dentro de las instituciones científicas victorianas. Hay dos razones para esto: una, haber sido ubicado como un personaje secundario dentro de la historia de la ciencia, siempre a la sombra de Charles Darwin, además de que, desde una mirada anacrónica, su involucramiento con el espiritismo todavía provoca reacciones adversas hacia el conjunto de sus ideas. Y segunda, es notable cómo el propio Wallace contribuyó a oscurecer sus propias aportaciones, en la medida en que ni en su autobiografía -en ninguna de las dos ediciones- ni en su correspondencia hace referencia a su participación en instituciones académicas. 
Resaltar el papel que Wallace jugó en las instituciones victorianas sirve para replantear su importancia dentro de la vida intelectual victoriana, y es que su labor no se quedó en ser un participante más, sino que fue el reconocimiento a su trabajo lo que le permitió incluso llegar a ser presidente de departamentos y secciones en la British Association for the Advancement of Science (BAAS), la agrupación de científicos más poderosa en el medio británico del siglo XIX, junto a la más que conocida Royal Society. Como aclaración, y a pesar de esa importancia, BAAS es una asociación que ha sido poco valorada en términos históricos y, aunque se le suele reconocer dentro de los recuentos de la ciencia victoriana (Lightman, 2019, p. 27), son pocos los trabajos que se han centrado en comprender el impacto que tuvo BAAS en la ciencia de la época (Basalla, Coleman y Kargon, 1970; Rodríguez Caso, 2014; Withers, 2010).

A su regreso a Inglaterra en 1862, al finalizar su viaje de ocho años al archipiélago Malayo, Wallace estaba interesado en involucrarse en la vida académica de Londres, para lo que se volvió un ávido asistente a reuniones de sociedades como la Ethnological Society of London (ESL) y la Anthropological Society of London $(A S L))^{3}$. Fue electo como miembro de ESL el 12 de junio de 1866, al parecer desencantado por las políticas defendidas por los dirigentes de ASL, abiertamente en favor de la esclavitud. A pesar de tal desencanto, su asistencia a las reuniones de ASL fue constante ${ }^{4}$, al grado que, como se ha dicho, una de sus más importantes contribuciones a las discusiones sobre la evolución humana se dio en la forma de una presentación, el $1 .^{\circ}$ de marzo de 1864, que a la postre sería la primera aplicación de la teoría de la selección natural al caso del ser humano, "The Origin of Human Races and the Antiquity of Man Deduced From the Theory of «Natural Selection»". En términos generales, Wallace buscaba con este trabajo conciliar las dos posturas dominantes sobre el origen de la humanidad entre los interesados en las ciencias del hombre, el monogenismo (un origen único) y el poligenismo (diversos orígenes). En sus propias palabras:

It is my wish to show how the two opposing views can be combined so as to eliminate the error and retain the truth in each, and it is by means of Mr. Darwin's celebrated theory of "Natural Selection" that I hope to do this, and thus to harmonize the conflicting theories of modern anthropologists" (Wallace, 1864, pp. clviii-clix).

Más adelante, se profundizará en los argumentos de este artículo.
Su papel dentro de BAAS merece una mención especial. Elegido miembro en 1863, realizó presentaciones sobre temas antropológicos en ese mismo año, "On the Varieties of Men in the Malay Archipelago", y en 1864, "On the Progress of Civilization in Northern Celebes". Sin embargo, el momento más relevante para Wallace dentro de su estancia en BAAS fue una ocasión que refleja la importancia que tenía dentro de la comunidad científica victoriana: su elección como el primer presidente de un espacio dedicado exclusivamente al estudio integral del ser humano, un departamento de antropología. ¿Por qué Wallace fue elegido para ese puesto, siendo que habría otros naturalistas mucho más cualificados por sus aportaciones a las "ciencias del hombre"? ${ }^{5}$ Son dos las respuestas: primero, el reconocimiento que ya tenía Wallace dentro del contexto victoriano, no solamente por su papel de codescubridor de la teoría de la selección natural, sino también por sus contribuciones en particular a la antropología. Y segundo, su carácter lo suficientemente amable para contentar a los dos grupos en pugna, ESL y ASL (Rodríguez Caso, 2018, p. 181).

Hablar de Wallace como presidente del nuevo departamento obliga también a recordar su particular y breve discurso inaugural, en el cual planteaba su visión sobre el quehacer antropológico, y de manera paralela hacía un listado de las diferentes profesiones y disciplinas que a su juicio formaban parte de tal quehacer, como la fisiología, la anatomía, la filología, la historia, la geología, la geografía e incluso la frenología:

Anthropology is the science which contemplates man under all his varied aspects (as an animal, and as a moral and intellectual being) in his relations to lower organisms, to his fellow men, and to the universe. The anthropologist seeks to collect together and systematize the facts and the laws which have been brought to light by all those branches of study which, directly or indirectly, have man for their object (Wallace, 1867, p. 93).

Sin ser necesariamente original, dado que definiciones similares en las que se hacía hincapié a la búsqueda del conocimiento integral del ser humano habían sido ya utilizadas por personajes como el médico francés Paul Broca o el foniatra británico James Hunt, lo dicho por Wallace buscaba, por un lado, establecer las bases a partir de las que todos aquellos interesados en las "ciencias del hombre" tuvieran cabida, en la medida en que el estudio del ser humano implicaba entender su complejidad en su conjunto. Por otro lado, es importante reconocer que el propio Wallace 
entendía al ser humano primero que nada como un animal, y en ese sentido relacionado con otros organismos, una postura que llama la atención si se considera que todavía en la década de 1860 la influencia de la teología natural, como el marco explicativo de la ciencia británica, era muy profunda. Pero al mismo tiempo, la postura de Wallace refleja su involucramiento en el movimiento del naturalismo científico, esto es, un conjunto de doctrinas que tenían "a commitment to the methods of the natural sciences and to the reliability of the knowledge generated by those methods" (Harrison, 2019, p. 1), una propuesta que buscaba explicar la naturaleza en términos estrictamente naturales, y alejados de explicaciones religiosas $^{6}$. Sin embargo, como se verá más adelante, existe un consenso en cuanto a que Wallace transitó de una defensa frontal del naturalismo científico hacia una visión evolutiva teleológica, con fuertes rasgos teístas ${ }^{7}$ (Fichman, 2004, pp. 283-321). Autores como Charles Smith proponen que Wallace defendió una visión no necesariamente teleológica, al menos en el sentido de entenderla estrictamente a partir de causas finales, sino que era más cercano a un "equilibrio general de fuerzas" inspirado en las ideas de Alexander von Humboldt (1769-1859) (Smith, 2019, pp. 41-66).

La participación institucional de Wallace no fue posiblemente tan significativa como en el caso de otros personajes victorianos pero, por lo menos para el caso de las ciencias del hombre, resultó de particular importancia, al fungir el papel de un mediador que permitió la unión de los diversos intereses científicos y naturalistas alrededor del ser humano. En la siguiente sección, se profundizará en algunos de esos intereses antropológicos que permitieron que Wallace formara parte de tan distinguida comunidad.

\section{CONTRIBUCIONES A LA ANTROPOLOGÍA FÍSICA}

\section{a. Origen de las razas humanas}

Como se mencionó anteriormente, el interés inicial de Wallace por entender la transmutación de las especies, y en especial del ser humano, a partir de la lectura de Vestiges, derivó en que muchos de sus escritos buscaran explicar diferentes aspectos del ser humano, como fue el origen y diversidad de los diferentes grupos humanos. Particularmente como resultado de su viaje al archipiélago malayo, logró reunir evidencias a partir de las cuales sustentar esos escritos. Uno de los temas por los que inicialmente Wallace participó de las discusiones antropológicas fue el del origen de la humanidad, un tema que estaba en su apogeo du- rante la década de 1860 en Inglaterra, de la mano de publicaciones tan relevantes como Antiquity of Man de Charles Lyell, y Man's Place in Nature de Thomas H. Huxley, ambos publicados en 1863. Parte de la discusión que se estableció a partir de ese momento, fue determinar no solamente el origen (u orígenes) de los seres humanos sino sobre todo su antigüedad. Esta discusión debe apreciarse dentro del contexto del surgimiento y consolidación del naturalismo científico, dado que una de las premisas bajo las que el mismo Wallace planteó sus argumentos -por lo menos en esa época-fue precisamente la del naturalismo.

Wallace en esta época, a mediados de 1860 , no proporcionó una fecha concreta para el origen de la humanidad, pero para él era claro que:

We can with tolerable certainty affirm that man must have inhabited the earth a thousand centuries ago, [...] We know positively that he was contemporaneous with many now extinct animals, and has survived changes of the earth's surface fifty or a hundred times greater than any that have occurred during the historical period; but we cannot place any definite limit to the number of species he may have outlived, or to the amount of terrestrial change he may have witnessed (Wallace, 1864, p. clviii).

A partir de tener claro que el ser humano ha estado presente en la vida del planeta por una gran cantidad de tiempo, ubicó su origen en la época terciaria, con la firme certeza de que el origen de los seres humanos (y su posible relación con los orangutanes) estaba en el sur de Asia, sin dejar de reconocer la importancia que podría tener África en un futuro:

In Murchison's address to the Geographical Society just delivered he points out Africa as being the oldest existing land. He says there is no evidence of its having been ever submerged during the tertiary epoch. Here, then, is evidently the place to find early man. I hope something good may be found in Borneo, and that then means may be found to explore the still more promising regions of tropical Africa, for we can expect nothing of man very early in Europe (Marchant, 1916, p. 159)

Esta postura sufrió pocos cambios con el paso de los años, y mucho tuvo que ver la reflexión que hizo Wallace sobre el papel de las condiciones ambientales, tanto pasadas como presentes, en la aparición de las primeras formas distintivamente humanas, al señalar que actualmente los primates se encontraban exclusivamente en regiones cercanas al ecuador, como el archipiélago malayo y África occidental, por lo que era 
de esperar que en esas mismas regiones se encontraran formas ancestrales, y como podemos ver resumido en su participación como Presidente de la Sección de Biología de BAAS, en 1876 señala:

But this objection is hardly valid, because existing anthropoid apes are wholly dependent on a perennial supply of easily accessible fruits, which is only found near the equator, while not only had the south of Europe an almost tropical climate in Miocene times, but we must suppose even the earliest ancestors of man to have been terrestrial and omnivorous, since it must have taken ages of slow modification to have produced the perfectly erect form, the short arms, and the wholly non-prehensile foot, which so strongly differentiate man from the arboreal apes [...] The conclusion which I think we must arrive at is, that if man has been developed from a common ancestor, with all existing apes, and by no other agencies than such as have affected their development, the he must have existed, in something approaching his present form, during the tertiary period - and not merely existed, but predominated in numbers, wherever suitable conditions prevailed. (Wallace, 1876, p. 114. Cursiva como en el original).

Años después, concluiría que, dadas las evidencias fósiles disponibles, así como los desarrollos culturales, el lugar más apropiado para buscar el origen de la humanidad debía ser el continente euroasiático, en algún punto entre el Mioceno tardío y el Plioceno temprano (Wallace, 1889, pp. 459-461). La ubicación temporal del origen de la humanidad respondió a la consecuencia de considerar que la mente humana proporcionaba ventajas adaptativas, como era la posibilidad de cambiar su ambiente a través de medios artificiales o culturales. Esta posición encuentra continuidad hoy en día con las discusiones particularmente de antropólogos y biólogos sobre la continuidad entre lo que se conoce como evolución biológica y evolución cultural. Conviene matizar que, aunque el estudio de la cultura se remonta a la llustración (Harris, 2017, pp. 588-589), fue en el siglo XIX cuando gracias a autores como el antropólogo británico Edward B. Tylor (18321917) con Primitive Culture (1871) y al antropólogo estadounidense Lewis Henry Morgan (1818-1881) con Ancient Society (1877) se sentaron las bases del evolucionismo sociocultural (Harris, 2017, pp. 589-590; Stocking, 1987, pp. 156-164). En el siglo XX, autores como el arqueólogo australiano Vere Gordon Childe (1892-1957) popularizaron el estudio del ser humano tanto en términos de evolución biológica como posteriormente cultural, a través de obras como What
Happened in History (1942). De igual forma, el trabajo del antropólogo germano-estadounidense Franz Boas (1858-1942) y de sus alumnos Margaret Mead (19011978), Alfred L. Kroeber (1876-1960) y Ruth Benedict (1887-1948) fueron vitales en la consolidación de los estudios culturales (Harris, 2017, p. 596). El estudio tanto de la evolución biológica como de la evolución cultural, a partir de esas categorías, se impuso desde la década de 1960 en adelante, un debate que se mantiene con gran fuerza en la actualidad.

Estos ejemplos sirven para hacer notar el claro interés de Wallace en la discusión sobre los orígenes, y en un continuo involucramiento en las discusiones que había en su época. Es notable ver cómo hace continuas referencias a personajes conocidos, como el propio Darwin, pero también a Huxley y a George Jackson Mivart (1827-1900) ${ }^{9}$, lo que habla de un personaje que estuvo mucho más involucrado en las discusiones científicas de la época de lo que se suele aceptar. Estas discusiones fueron abordadas por Wallace desde el naturalismo científico, y es que, aunque se reconoce que su transición hacia la teleología se dio a partir de la década de 1870 (Fichman, 2004 p. 283), veremos que fue con sus últimas publicaciones cuando consolidó su defensa del principio antrópico, y con ello su teleología evolutiva.

\section{b. Desarrollo de características distintivas del ser humano}

Más allá de ubicar el origen del ser humano a partir de la propuesta que había presentado junto a Darwin, la concepción que Wallace tuvo sobre los alcances de la selección natural se desarrolló siempre desde una perspectiva marcadamente utilitarista, es decir, "that selection could preserve only what was useful in the struggle for existence" (Radick, 2007, p. 34), y es que como lo plantea en su artículo de 1864:

If the views I have here endeavoured to sustain have any foundation, they give us a new argument for placing man apart, as not only the head and culminating point of the grand series of organic nature, but as in some degree a new and distinct order of being. From those infinitely remote ages, when the first rudiments of organic life appeared upon the earth, every plant, and every animal has been subject to one great law of physical change. [...] No living thing could escape this law of its being; none could remain unchanged and live, amid the universal change around it (Wallace, 1864, p. clxvii).

Es claro que la visión que Wallace defiende aquí es profundamente teleológica y antropocéntrica, al no- 
tar que la evolución, a través de la selección natural, proporciona un marco explicativo bajo el que el ser humano es un producto diferente al resto. Años después, Wallace afirmará que:

The most important difference between man and such of the lower animals as most nearly approach him is undoubtedly in the bulk and development of his brain, as indicated by the form and capacity of the cranium (Wallace, 1876, p. 113).

Incluso planteaba que los recientes descubrimientos de cráneos fósiles como los de Engis y Cro-Magnon permitían aún hablar de características humanas distintivas, que no solamente se apreciaban en el nivel físico, sino que eran extensivas a aspectos culturales, como el desarrollo de las pinturas rupestres o la creación de herramientas. Ese antropocentrismo, de la mano de ideas sobre el propósito del universo, culminaría en Man's Place in the Universe: A Study of the Results of Scientific Research in Relation to the Unity or Plurality of Worlds (1903), una obra que "sheds unique light on Wallace's anthropocentric world view, and illuminates his entire career, including his belief that the human mind must be set off from animals in the evolutionary process" (Dick, 2008, p. 320).

Ese reconocimiento a la importancia de la mente sobre cualquier otra característica humana lo va a reafirmar continuamente, pero con ese guiño a la naturaleza animal de los seres humanos:

Here, then, we see the true grandeur and dignity of man [the human mind]. On this view of his special attributes, we may admit that even those who claim for him a position as an order, a class, or a sub-kingdom by himself, have some reason on their side. $\mathrm{He}$ is, indeed, a being apart, since he is not influenced by the great laws which irresistibly modify all other organic beings. Nay more; this victory which he has gained for himself gives him a directing influence over other existences (Wallace, 1864, p. clxviii).

Una de esas características que Wallace explicó con mayor detalle es la del lenguaje. La postura que defendió es la llamada teoría gestual, que tuvo gran influencia en los siglos XVIII y XIX. Como señala el antropólogo estadounidense Gordon Hewes, fue a partir de una reseña que Wallace hizo sobre los planteamientos de Edward B. Tylor en Anthropology: an introduction to the study of man and Civilization (1881) sobre la importancia de los gestos faciales como los que permitieron el paso de los signos manuales a los vocales, que hizo la teoría gestual mucho más explícita (Hewes, 1976, p. 486). En trabajos previos Wallace partía de considerar las limitaciones de la selección natural, en la medida en que "could only have endowed the savage with a brain a little superior to that of an ape, whereas he actually possesses one but very little inferior to that of the average members of our learned societies" ([Wallace], 1869a, p. 392) ${ }^{10}$. Esta afirmación ha llevado en tiempos recientes a autores interesados en la evolución del lenguaje a concebir el problema de Wallace (Bickerton, 2014, p. 1), en la medida en que fue el primero en plantear que "[i]f evolution was a gradual process, and natural selection responded only to the demands placed on animals by their environment, then humans should have had a brain 'little superior to that of an ape'" (Bickerton, 2014, p. 2). La respuesta parece estar en que "extraordinary competences required extraordinary selective pressures, best understood by searching for analogies far away in phylogenetic space" (Levinson, 2014, p. 1458), es decir, si se parte de considerar al ser humano como un ente totalmente diferente a otros, a pesar de tener un posible origen común, es en la búsqueda de explicaciones concretas para su naturaleza donde se podrán encontrar respuestas.

El punto central del argumento de Wallace es que, al comparar diferentes lenguas se puede observar que tienen una similitud en la forma en que se pronuncian, derivada de un notorio parecido en los movimientos producidos por la boca, que va más allá de un simple proceso de imitación:

In our own language, and probably in all others, a considerable number of the most familiar words are so constructed as to proclaim their meaning more or less distinctly, sometimes by means of imitative sounds, but also, in a large number of cases, by the shape or the movements of the various parts of the mouth used in pronouncing them, and by peculiarities in breathing or in vocalization, which may express a meaning quite independent of mere sound-imitation (Wallace, 1895, p. 528).

Esta afirmación se basó tanto en el interés de Wallace en la lingüística comparada ${ }^{11}$ como en sus observaciones de los diferentes grupos humanos que conoció durante sus viajes.

Al final, para Wallace existe un marcado determinismo entre el desarrollo físico de ciertas características y el respectivo sonido (o conjunto de sonidos) que pueden mostrar, y esto es común en diferentes grupos humanos:

These mouth-gestures, [...] necessarily lead to distinct classes of sounds; and thus there arose from 
the very beginnings of articulate speech, the use of characteristic sounds to express certain groups of motions, actions, and sensations which we are still able to detect even in our highly-developed language, and the more important of which I have here attempted to define and illustrate (Wallace, 1895, p. 543).

La transición del naturalismo hacia una teleología teísta siempre tuvo como eje la evolución. En cierta forma, con el paso del tiempo Wallace amplió el marco de su explicación evolutiva, hasta llegar a un teísmo pragmático (Fichman, 2004, pp. 292-293), que le permitió conjuntar de manera coherente, por lo menos para él, sus diversos intereses, tanto académicos como personales. Aunque, como hemos visto ya, Wallace centró buena parte de sus intereses antropológicos en el ser humano y sus particularidades, veremos a continuación que también se ocupó de otros primates también en sus trabajos como naturalista.

\section{c. Relación evolutiva entre humanos y primates}

La obra de Chambers Vestiges proporcionó a Wallace material para considerar otros temas, además de impulsarlo a buscar un mecanismo que explicara la transmutación de las especies, y sobre todo del ser humano. Una de las hipótesis planteadas en esa obra era que el origen de los seres humanos, relacionados a su vez con los Cuadrumana ${ }^{12}$, y en especial con los orangutanes, se encontraba en esa región: “[...] we should expect man to have originated where the highest species of the quadrumana are to be found. Now these are unquestionably found in the Indian Archipelago" ([Chambers], 1844, p. 296). Esto explica en parte el interés de Wallace por visitar la zona, además de que, como en el caso de su anterior viaje al Amazonas, había dos justificaciones: buscar el mecanismo para explicar la transmutación, y conseguir ejemplares de diferentes organismos para venderlos a coleccionistas privados y a museos en Europa:

One of my chief objects in coming to stay at Simunjon was to see the Orang-utan (or great manlike ape of Borneo) in his native haunts, to study his habits, and obtain good specimens of the different varieties and species of both sexes, and of the adult and young animals. In all these objects I succeeded beyond my expectations, and will now give some account of my experience in hunting the Orangutan, or "Mias," as it is called by the natives; and as this name is short, and easily pronounced, I shall generally use it in preference to Simia satyrus, or Orang-utan. (Wallace, 1869b, pp. 61-62).

Recientemente, se ha propuesto:
We see nothing about a search for human origins in Wallace's observations of orangutans. Indeed, although he was actively pursuing his private interests in evolutionary theory at exactly this time, his notes on orangutans contain no mention of human origins or evolution" (van Wyhe y Kjærgaard, 2015, p. 60).

Sin embargo, durante la década de 1850 Wallace publicó diversos trabajos que buscaron reflejar diversos aspectos de los orangutanes, como el desarrollo de sus diferentes etapas de vida, aspectos conductuales y de hábitos producto de observaciones realizadas en el campo, y fue como resultado de comparaciones morfológicas entre primates y seres humanos como concibió las posibles relaciones entre seres humanos y orangutanes, en un sentido que claramente se puede interpretar como de descendencia a partir de un origen común:

And when we consider that almost all other animals have in previous ages been represented by allied, yet distinct forms,--that the bears and tigers, the deer, the horses, and cattle of the tertiary period were distinct from those which now exist, with what intense interest, with what anxious expectation must we look forward to the time when the progress of civilization in those hitherto wild countries may lay open the monuments of a former world, and enable us to ascertain approximately the period when the present species of Orangs first made their appearance, and perhaps prove the former existence of allied species still more gigantic in their dimensions, and more or less human in their form and structure! (Wallace, 1856b, p. 31)

Y de igual forma, cuando habla de las similitudes morfológicas entre primates:

One cannot help speculating on a former condition of this part of the world which should give a wider range to these strange creatures, which at once resemble and mock the "human form divine", which so closely approach us in structure, and yet differ so widely from us in many points of their external form (Wallace, 1856b, p. 31).

Es cierto que, a pesar de estos dichos, se coincide con lo indicado por James Costa en el sentido de que Wallace, en sus inicios como naturalista, daba explicaciones poco detalladas sobre sus observaciones, que se pueden interpretar como un "silencio ensordecedor" (Costa, 2014, p. 34). Años después, insistiría en reconocer el sur de Asia como el origen no solamente de los seres humanos, sino de los primates en general: 
When we consider, further, that almost all other animals have in earlier ages been represented by allied yet distinct forms - that, in the latter part of the tertiary period, Europe was inhabited by bears, deer, wolves, and cats; Australia by kangaroos and other marsupials; South America by gigantic sloths and anteaters; all different from any now existing, though intimately allied to them-we have every reason to believe that the Orang-utan, the Chimpanzee, and the Gorilla have also had their forerunners. With what interest must every naturalist look forward to the time when the caves and tertiary deposits of the tropics may be thoroughly examined, and the past history and earliest appearance of the great man-like apes be at length made known (Wallace, 1869b, p. 96).

Este tipo de afirmaciones sirvieron para que, años después, el anatomista holandés Eugène Dubois (1858-1940) se decidiera a buscar restos fósiles humanos en la región descrita por Wallace, que resultaría en el descubrimiento del Pithecanthropus erectus, hoy en día conocido como Homo erectus (Theunissen, 1989, p. 34). Una interesante muestra de la influencia que tuvo Wallace dentro de la investigación antropológica.

\section{CONCLUSIONES}

Un año antes de la publicación de The Descent of Man de Darwin, en 1870, Wallace decía:

[...] if in proportion as man's social, moral, and intellectual faculties became developed, his physical structure would cease to be affected by the operation of "natural selection," we have a most important clue to the origin of races" (Wallace, 1870, p. 319).
Su profunda convicción sobre la importancia de la selección natural ha llevado a que se le considere un hiperseleccionista, pero es claro que resulta llamativo que concibió límites a su acción. Aunque se suele afirmar que el espiritismo le restó legitimidad a su posición como científico, como se puede ver a lo largo de este texto, Wallace partió inicialmente de una defensa de explicaciones naturalistas y fue en el utilitarismo en donde encontró la base explicativa para considerar que, en la evolución humana, había un punto en que la selección no podía funcionar.

Un aspecto importante que destacar es que, a pesar de su interés en temas sobre antropología física, y que con el paso de los años su postura fue derivando hacia una teleología teísta evolutiva, se pueden ubicar explicaciones de particular importancia en el ámbito antropológico, sobre todo del siglo XIX, como aplicar la selección natural al origen de los seres humanos, plantear la importancia del aspecto cultural en el desarrollo de la naturaleza humana y participar activamente en discusiones que sirvieron para moldear la antropología en términos institucionales.

A pesar de ser un hombre con numerosos intereses, la historización de los seres humanos fue la guía principal de su labor como naturalista, y esa idea lo llevó a buscar entender lo humano desde las más diversas perspectivas, una posición que podemos ver en su propia definición de antropología, pero sobre todo en su papel como defensor de la selección natural.

\section{AGRADECIMIENTOS}

Agradezco al Programa de Becas Posdoctorales de la Universidad Nacional Autónoma de México por el apoyo brindado para realizar esta investigación.

\section{NOTAS}

1. Darwin menciona en su carta que el artículo apareció en Anthropological Review, que también pertenecía a ASL, pero en realidad se publicó en Journal of the Anthropological Society of London.

2. La expresión tiempo profundo se refiere al tiempo geológico, y en términos modernos el concepto fue desarrollado por el geólogo escocés James Hutton (17261797).

3. ESL fue fundada en 1842 , mientras que ASL apareció en 1863 como una escisión de la primera. En términos generales, ESL se caracterizó por defender el monogenismo y la evolución, mientras que ASL promovía el poligenismo y estaba en contra de la evolución.
4. Una particularidad de las reuniones de ambas asociaciones es que, a pesar de la separación, se daban en el mismo espacio, con una hora de diferencia, en 4 St Martin's Place, muy cerca del National Portrait Gallery, enfrente de Trafalgar Square.

5. Esta expresión, acuñada por David Hume, sobre todo en la tradición británica ha servido para caracterizar de manera más neutral al conjunto de disciplinas que estudian diferentes aspectos del ser humano.

6. El involucramiento de Wallace con el naturalismo científico fue un tanto efimero, ya que en 1865 conoció el espiritismo, que influyó definitivamente en su paso hacia una teleología evolutiva teísta, como lo ha planteado Martin Fichman (2004).

7. Como aclaración, el teísmo defendido por Wallace no era cristiano, ya que se alejó de las instituciones religiosas desde muy joven. Tampoco era un "espiritista teísta", por lo menos por haberse alejado de los cánones espiritistas más generales. El teísmo para Wallace "constituted a legitimate component of a valid and inclusive system of investigating nature", es decir, la Inteligencia Suprema era parte de la naturaleza, y por lo tanto parte de las explicaciones naturales (véase Fichman, 2004, pp. 286-291). 
8. Carta enviada por Wallace a Charles Darwin el 29 de mayo de 1864. Se refiere al discurso de Sir Roderick Impey Murchison (1792-1871), reconocido geólogo escocés que entre otras aportaciones estableció los sistemas Silúrico y Devónico.

9. Biólogo británico, alumno de Thomas Huxley, conocido por sus trabajos en zoología de mamíferos y por su ardua defensa en reconciliar el catolicismo con la teoría evolutiva.

\section{BIBLIOGRAFÍA}

Álvarez Muñoz, E. (2004). Filosofía de las ciencias de la tierra. El cierre categorial de la geología. Oviedo: Pentalfa.

Basalla, G., Coleman, W. y Kargon, R. H. (eds.). (1970). Victorian Science: A Selfportrait from the Presidential Addresses of the British Association for the Advancement of Science. Garden City: Anchor Books.

Bickerton, D. (2014). More than Nature Needs. Cambridge, Londres: Harvard University Press. https://doi. org/10.4159/9780674728523

Bravo, M. T. (1996). Ethnological Encounters. En Jardine, N., Secord, J. A. y Spary, E. C. (eds.). Cultures in Natural History. Cambridge: Cambridge University Press, pp. 338-357.

Brotman, C. (2001). Alfred Wallace and the Anthropology of Sound in Victorian Culture. Endeavour, 25 (4), pp. 144147. https://doi.org/10.1016/S01609327(00)01385-5

[Chambers, R.] (1844). Vestiges of the $\mathrm{Na}$ tural History of Creation. Londres: John Churchill, Princes Street, Soho.

Costa, J. T. (2014). Wallace, Darwin, and the Origin of Species. Cambridge, Londres: Harvard University Press. https://doi. org/10.4159/harvard.9780674416468

Darwin, C. (1859). On the origin of species by means of natural selection, or the preservation of favoured races in the struggle for life. Londres: John Murray. https://doi.org/10.5962/bhl.title.68064

Davis, R. W. (ed.) (1990). Carr, E. H. What is History? The George Macaulay Trevelyan Lectures Delivered in the University of Cambridge January-March 1961

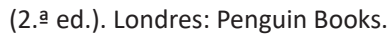

Dick, S. J. (2008). The Universe and Alfred Russel Wallace. En Smith, C. H. y BeccaIoni, G. (eds.). Natural Selection and Be-
10. Esta reseña de la obra de Lyell fue originalmente publicada de manera anónima y es mayoritariamente reconocida como el trabajo donde Wallace establece sus principales objeciones al papel de la selección natural respecto a la mente humana.

11. Como, por ejemplo, el apéndice que desarrolló sobre diversos lenguajes nativos del archipiélago Malayo (véase Wallace, 1869b, pp. 465-502).

yond: The Intellectual Legacy of Alfred Russel Wallace. Oxford: Oxford University Press, pp. 320-340.

Eiseley, L. C. (1959). Darwin's century: Evolution and the men who discovered it. Londres: The Scientific Book Guild.

Ellen, R. (2011). The place of the eolithic controversy in the anthropology of Alfred Russel Wallace. The Linnean, 27 (1), pp. 22-33

Fichman, M. (2004). An Elusive Victorian: The Evolution of Alfred Russel Wallace. Chicago, Londres: University of Chicago Press. https://doi.org/10.7208/chicago/9780226246154.001.0001

Harris, M. (2017). Antropología cultural. Madrid: Alianza Editorial.

Harrison, P. y Roberts, J. H. (eds.) (2019). Science Without God? Rethinking the History of Scientific $\mathrm{Na}$ turalism. Oxford: Oxford University Press. https://doi.org/10.1093/ oso/9780198834588.001.0001

Hewes, G. W. (1976). The Current Status of the Gestural Theory of Language Origin. New York Academy Sciences Annals, 280, pp. 482-504. https:// doi.org/10.1111/j.1749-6632.1976. tb25512.x

Hodge, M. J. S. (2015). Wallace y Darwin: los dos primeros darwinistas. En Rodríguez, J. M., Ruiz, R., Oliver, C. y Abarca, C. (eds.) Alfred R. Wallace, a cien años de su muerte. México: Universidad Nacional Autónoma de México, pp. 59-67.

Kottler, M. J. (1974). Alfred Russel Wallace, the Origin of Man, and Spiritualism. Isis, 65 (2), pp. 145-192. https://doi. org/10.1086/351256

Levinson, S. (2014). Language and Wallace's Problem. Science, 344 (6191), pp. 14581459. https://doi.org/10.1126/science. 1252988
12. División actualmente obsoleta, propuesta por el médico y naturalista alemán Johann Friedrich Blumenbach (1752-1840) para designar a los primates de "cuatro manos", en contraposición con los Bimana, que servía para designar a los seres humanos.

Lightman, B. (2019). The Evolution of the Scientific Disciplines. En B. Lightman, B. y Zon, B. (eds.). Victorian Culture and the Origin of Disciplines. New York, Oxon: Routledge, pp. 21-40. https://doi. org/10.4324/9780429277139-2

Marchant, J. (ed.). (1916). Alfred Russel Wallace, Letters and Reminiscences (vols. 1-2). Londres: Cassell.

McKinney, H. L. (1969). Wallace's Earliest Observations on Evolution: 28 December 1845. Isis, 60 (3), pp. 370-373. https://doi.org/10.1086/350505

McKinney, H. L. (1972). Wallace and Natural Selection. New Haven: Yale University Press.

Radick, G. (2007). The Simian Tongue: The Long Debate About Animal Language. Chicago, Londres: University of Chicago Press.

Rodriguez Caso, J. M. (2014). Anthropology in Transition: A Study of the Sciences of Man at the British Association for the Advancement of Science, 1866-1870 [Tesis doctoral inédita]. University of Leeds.

Rodríguez Caso, J. M. (2015). Estudiar al hombre en sus más variados aspectos: A. R. Wallace, antropólogo. En Rodríguez, J. M., Ruiz, R., Oliver, C. y Abarca, C. (eds.) Alfred R. Wallace, a cien años de su muerte. México: Universidad Nacional Autónoma de México, pp. 95-102.

Rodríguez Caso, J. M. (2018). Institucionalización de la antropología en las reuniones de la British Association for the Advancement of Science (BAAS), 18631870. Cuicuilco. Revista de Ciencias Antropológicas, 25 (73), pp. 167-188.

Rodríguez Caso, J. M. (2019). Historización de la naturaleza del ser humano en $\mathrm{A}$. R. Wallace. Saberes. Revista de historia de las ciencias y las humanidades, 2 (5), pp. 141-171. 
Secord, J. A. (2000). Victorian Sensation: The Extraordinary Publication, Reception, and Secret Authorship of Vestiges of the Natural History of Creation. Chicago: University of Chicago Press. https://doi.org/10.7208/chicago/9780226158259.001.0001

Smith, R. (1972). Alfred Russel Wallace: Philosophy of Nature and Man. The British Journal for the History of Science, 6 (2), pp. 177-199. https://doi.org/10.1017/ S0007087400012279

Smith, C. H. (2019). Wallace and the "Preter-normal”. En Smith, C. H,. Costa, J. T. y Collard, D. (eds.). An Alfred Russel Wallace Companion. Chicago, Londres: University of Chicago Press, pp. 41-66. https://doi.org/10.7208/chicago/9780226622248.003.0003

Stocking, G. W. (1987). Victorian Anthropology. Nueva York: Free Press.

Theunissen, B. (1989). Eugène Dubois and the Ape-Man from Java: The History of the First 'Missing Link' and Its Discoverer. Dordrecht, Boston, Londres: Springer Science \& Business Media.

Vetter, J. (2009). The Unmaking of an Anthropologist: Wallace Returns from the Field, 1862-70. Notes and Records of the Royal Society, 64 (1), pp. 25-42. https://doi.org/10.1098/ rsnr.2009.0013

Wallace, A. R. (1856a) Notes of a Journey Up the Sadong River, North-West Borneo. Proceedings of the Royal Geographical Society of London, 6, pp. 193-205.
Wallace, A. R. (1856b). On the Habits of the Orang-Utan of Borneo. Annals \& Magazine of Natural History, 18 (103), pp. 26-32. https://doi. org/10.1080/00222935608697572

Wallace, A. R. (1860). Notes of a Voyage to New Guinea. Journal of the Royal Geographical Society, 30, pp. 172-177. https://doi.org/10.2307/1798299

Wallace, A. R. (1864). The Origin of Human Races and the Antiquity of Man Deduced from the Theory of «Natural Selection». Journal of the Anthropological Society of London, 2, pp. clviii-clxxxvii. https://doi.org/10.2307/3025211

Wallace, A. R. (1867). Anthropology. En British Association for the Advancement of Science. Report of the Meeting of the British Association for the Advancement of Science. Londres: John Murray, pp. 93-94.

[Wallace, A. R.] (1869a). Sir Charles Lyell on Geological Climates and the Origin of Species. Quarterly Review, 126 (252), pp. 359-394.

Wallace, A. R. (1869b). The Malay Archipelago: The Land of the Orang-utan, and the Bird of Paradise. A Narrative of Travel, with Studies of Man and Nature. Londres: Macmillan \& Co. https://doi. org/10.5962/bhl.title.134833

Wallace, A. R. (1870). Contributions to the Theory of Natural Selection: A Series of Essays. Londres, Nueva York: Macmillan \& Co. https://doi.org/10.5962/bhl.title. 1254
Wallace, A. R. (1876). Biology. En British Association for the Advancement of Science. Report of the Meeting of the British Association for the Advancement of Science. Londres: John Murray, pp. 100-119.

Wallace, A. R. (1889). Darwinism: An Exposition of the Theory of Natural Selection. Londres: Macmillan \& Co.

Wallace, A. R. (1895). The Expressiveness of Speech, Or, Mouth-Gesture as a Factor in the Origin of Language. Fortnightly Review, 58 (346), pp. 528-543.

Wallace, A. R. (1905). My Life: A Record of Events and Opinions (vols. 1-2). Londres: Chapman \& Hall. https://doi. org/10.5962/bhl.title.96039

Withers, C. W. J. (2010). Geography and Science in Britain, 1831-1939: A Study of the British Association for the Advancement of Science. Manchester: Manchester University Press.

Wyhe, J. van y Kjærgaard, P. C. (2015). Going the Whole Orang: Darwin, Wallace and the Natural History of Orangutans. Studies in History and Philosophy of Science. Part C: Studies in History and Philosophy of Biological and Biomedical Sciences, 51, pp. 53-63. https://doi. org/10.1016/j.shpsc.2015.02.006

\section{Recursos en línea}

Carta de Darwin a Wallace, 26 enero 1870. En Darwin Correspondence Project, Letter no. 7086. [En línea]. Disponible en: http://www.darwinproject.ac.uk/DCPLETT-7086 